\title{
Venom gland transcriptomes of two elapid snakes (Bungarus multicinctus and Naja atra) and evolution of toxin genes
}

\author{
Yu Jiang ${ }^{1,2+}$, Yan $\mathrm{Li}^{3 \dagger}$, Wenhui Lee ${ }^{4 \dagger}$, Xun Xu', Yue Zhang ${ }^{1}$, Ruoping Zhao' ${ }^{1}$ Yun Zhang ${ }^{4^{*}}$, Wen Wang ${ }^{{ }^{*}}$
}

\begin{abstract}
Background: Kraits (genus Bungarus) and cobras (genus Naja) are two representative toxic genera of elapids in the old world. Although they are closely related genera and both of their venoms are very toxic, the compositions of their venoms are very different. To unveil their detailed venoms and their evolutionary patterns, we constructed venom gland cDNA libraries and genomic bacterial artificial chromosome (BAC) libraries for Bungarus multicinctus and Naja atra, respectively. We sequenced about 1500 cDNA clones for each of the venom CDNA libraries and screened BAC libraries of the two snakes by blot analysis using four kinds of toxin probes; i.e., three-finger toxin (3FTx), phospholipase A2 (PLA2), kunitz-type protease inhibitor (Kunitz), and natriuretic peptide (NP).

Results: In total, 1092 valid expressed sequences tags (ESTs) for B. multicinctus and 1166 ESTs for N. atra were generated. About $70 \%$ of these ESTs can be annotated as snake toxin transcripts. 3FTx (64.5\%) and $\beta$ bungarotoxin (25.1\%) comprise the main toxin classes in B. multicinctus, while 3FTx (95.8\%) is the dominant toxin in N. atra. We also observed several less abundant venom families in B. multicinctus and N. atra, such as PLA2, C-type lectins, and Kunitz. Peculiarly a cluster of NP precursors with tandem NPs was detected in B. multicinctus. A total of 71 positive toxin BAC clones in B. multicinctus and N. atra were identified using four kinds of toxin probes (3FTX, PLA2, Kunitz, and NP), among which 39 3FTx-postive BACs were sequenced to reveal gene structures of 3FTx toxin genes.

Conclusions: Based on the toxin ESTs and 3FTx gene sequences, the major components of B. multicinctus venom transcriptome are neurotoxins, including long chain alpha neurotoxins $(\alpha-n t x)$ and the recently originated $\beta$ bungarotoxin, whereas the $N$. atra venom transcriptome mainly contains 3FTxs with cytotoxicity and neurotoxicity (short chain $\alpha$-ntx). The data also revealed that tandem duplications contributed the most to the expansion of toxin multigene families. Analysis of nonsynonymous to synonymous nucleotide substitution rate ratios (dN/dS) indicates that not only multigene toxin families but also other less abundant toxins might have been under rapid diversifying evolution.
\end{abstract}

\section{Background}

Snake venoms comprise a diverse array of toxins that have a variety of biochemical and pharmacological functions and can be conveniently classified as hemotoxins and neurotoxins [1]. Recently, it has been documented

\footnotetext{
* Correspondence: zhangy@mail.kiz.ac.cn; wwang@mail.kiz.ac.cn

+ Contributed equally

'CAS-Max Planck Junior Research Group, State Key Laboratory of Genetic Resources and Evolution, Kunming Institute of Zoology, Chinese Academy of Sciences, Kunming, Yunnan 650223, China

${ }^{4}$ Biotoxin Units, Key Laboratory of Animal Models and Human Disease Mechanisms, Kunming Institute of Zoology, Chinese Academy of Sciences, Kunming, Yunnan 650223, China

Full list of author information is available at the end of the article
}

that most of the snake toxins were recruited or derived from normal body proteins in the common ancestor of venomous squamates (Toxicofera) or advanced snakes (Caenophidia) 100-200 million years ago (mya) [2-5]. Associated with the species radiation of advanced snakes in the Cenozoic era, a predator-prey arms race led to the explosive appearance of toxic arsenals, and typically, several toxin multigene families were generated by gene duplication, followed by fast diversification $[4,6]$. The birth-and-death model was proposed to explain the emergence of taxon-specific toxin groups [7]; i.e., new toxin genes consistently emerged through gene duplication with the divergence of taxa, but some toxin genes

\section{Biomed Central}


got deleted or were degenerated in other lineages. However, due to the low-depth sequencing of toxin transcripts for each species, fast evolution of toxin genes, and lack of genome sequences, the detailed elaboration of snake venom evolution is still unclear.

The Elapidae family (elapids), which has approximately 300 venomous snakes in 61 genera, is a monophyletic group among advanced snakes [8,9]. Several broad radiated lineages (diverged rapidly between around 31 and 26 mya, based on fossil records and molecular evidence [9]) have been identified within the Elapidae, including the Afro-Asian cobras, Oriental kraits, AsianAmerican corals, and Australian and marine snakes, which are well known to be the most toxic snakes in the world. So far, the gene expression profiles of venom glands from four species [10-12] have been reported using EST sequencing. However, the kraits (genus Bungarus) and cobras (genus Naja), as the most diverse and representative toxic elapids in the old world [7], lack genomic and venom EST data.

In the present study, we prepared cDNA libraries from the venom glands of the two representative old world elapid snakes, Bungarus multicinctus and Naja atra, and sequenced about 1500 clones for each library. We also constructed genomic bacterial artificial chromosome (BAC) libraries for the two snakes and conducted a screen for venom genes. Our results identified many new snake toxins, such as multiple groups of 3FTxs, some novel lectins, and a peculiar natriuretic peptide (NP), and revealed that toxin genes have experienced rapid evolution and gene expansion.

\section{Results and Discussion}

\section{Venom gland cDNA libraries and ESTs}

We constructed two cDNA libraries from the venom gland tissues of B. multicinctus and $N$. atra, respectively, and randomly selected 1441 and 1534 clones for sequencing. From these clones, we successfully obtained 1092 expressed sequences tags (ESTs) for B. multicinctus and 1166 ESTs for $N$. atra. Using CAP3 software with a cutoff of $95 \%$ identity [13], the B. multicinctus ESTs were grouped into 294 clusters (including 83 contigs assembled by two or more ESTs and 211 singlets), whereas the $N$. atra ESTs were grouped into 339 clusters (including 62 contigs and 277 singlets). Using BLAST for functional annotation, complete lists with putative gene identifications for these EST clusters are provided in Additional file 1. Most ESTs could be matched to reported snake toxins $(69.51 \%$ in $B$. multicinctus and $70.24 \%$ in $N$. atra). The toxin clusters showed notably high redundancy (14.32 ESTs/cluster in B. multicinctus, $12.41 \mathrm{ESTs} / \mathrm{cluster}$ in $N$. atra), in contrast to the low redundancy of non-toxin clusters $(1.40$ ESTs/cluster in B. multicinctus, $1.31 \mathrm{ESTs} /$ cluster in $N$. atra) (Table 1), indicating that the toxin genes were highly expressed.

In this study, we focused on the toxin ESTs; i.e., 26 contigs and 27 singlets in $\mathrm{B}$. multicinctus, and 18 contigs and 48 singlets in $N$. atra (Figure 1). Except the dominant 3FTx, we also observed eight less relatively abundant venom families both in B. multicinctus and $N$. atra: phospholipase A2 (PLA2), C-type lectins, kunitztype protease inhibitor (Kunitz), L-Amino oxidase (LAO), nerve growth factor (NGF), cysteine-rich secretory protein (CRSIP), and vespryn. However, NP, presynaptic $(\beta)$ bungarotoxin, prothrombin activator $(\mathrm{Pa})$, and acetylcholinesterase were only detected in B. multicinctus, while metalloproteinase and cystatin were only found in $N$. atra.

In snake venoms, 3FTx, PLA2, Kunitz, C-type lectins, and metalloproteinase have been reported to be the major multigene toxin families [6], and they comprised $97.1 \%$ in B. multicinctus and $98.0 \%$ in $N$. atra in our toxin EST data. We also identified a peculiar cluster of NP precursors with tandem NPs. Besides, $\beta$ bungarotoxin is a Bungarous-specific toxin, which is a heterodimeric protein complex of phospholipase A2 (A chain) and kunitz peptides (B chain) covalently linked by one disulphide bridge $[4,14]$. Here, we detected 7 clusters (108 ESTs) of A chain of $\beta$ bungarotoxin and 4 clusters (83 ESTs) of B chain as a major toxin in B. multicinctus (25.1\% of all toxin ESTs).

\section{Analysis of toxin genes Three-finger toxins (3FTXs)}

3FTxs are neurotoxins and can bind to very different neurological targets (neuronal, pre-synaptic, postsynaptic, and synaptic); some of them also have non-neurotoxic activities (cytotoxicity and platelet inhibition) $[4,5]$. Its family members have a similar structure, with three loops and four conserved disulfide bridges, and contain about 60-74 amino acid residues. The ancestral 3FTx was thought to have 10 cysteines and acquired some amelioration: deletion of the ancestral $C^{2}$ and $C^{3}$ cysteines potentiated alpha-neurotoxic activity and acquired additional newly evolved cysteines [7]. In 2003, Fry grouped 33 types of 3FTxs from 276 protein sequences, with a number of "orphan groups" of 3FTxs whose functional roles are not yet known [7]. Since then, many other kinds of 3FTxs have been successively detected $[12,15,16]$. So far, more than five hundred variant 3FTx sequences across the entire advanced snakes have been observed. A birth-and-death mode [7] and a mechanism of duplication and divergence [17], allowing venom snakes to adapt to a variety of prey, were proposed to explain the generation of the $3 \mathrm{ftx}$ superfamily. But, the evolutionary relationships of all kinds of 3FTxs are still uncertain, such as the notable alpha 
Table 1 Composition of ESTs obtained from B. multicinctus and N. atra venom glands

\begin{tabular}{llllll}
\hline & $\begin{array}{l}\text { Transcripts } \\
\text { category }\end{array}$ & $\begin{array}{l}\text { Number of } \\
\text { ESTs }\end{array}$ & $\begin{array}{l}\text { Number of } \\
\text { clusters }\end{array}$ & $\begin{array}{l}\text { Redundancy (clones/ } \\
\text { clusters) }\end{array}$ & $\begin{array}{l}\text { Representation over total clones } \\
\text { (\%) }\end{array}$ \\
\hline $\begin{array}{l}\text { B. } \\
\text { multicinctus }\end{array}$ & Toxins & 760 & 54 & 14.07 & 69.60 \\
& Non-toxins & 288 & 205 & 1.40 & 26.37 \\
& No hit & 44 & 35 & 1.26 & 4.03 \\
\hline N. atra & Toxins & 819 & 66 & 12.41 & 70.24 \\
& Non-toxins & 304 & 232 & 1.31 & 26.07 \\
& No hit & 43 & 41 & 1.05 & 3.69 \\
\hline
\end{tabular}

neurotoxic clades (short chain, long chain, kappa, and type III), which are not monophyletic, based on the phylogenetic tree of 3FTxs constructed by Fry [4]. It is in contrast to previous hypotheses that alpha neurotoxins $(\alpha$-ntx) diverged from a common ancestral gene that separated before the divergence of the elapid sub-family [18].

The analysis of the B. multicinctus 3FTxs revealed that 491 clones (64.5\% of all toxin ESTs) could be grouped into 12 contigs and 9 singlets, and a total of $785 \mathrm{~N}$. atra clones (95.8\% of all toxin ESTs) fell into 12 contigs and 35 singlets. We notice that all the contig sequences encoded full-length proteins, except two contigs (na009 with 3 clones, bm006 with 7 clones), which are lacking exon2, probably resulting from alternative splicing. Since 3FTxs are the dominant toxins in both $B$. multicinctus and $N$. atra and show a greater diversity of family members, we classified them basically based on the number of cysteines following the nomenclature of Fry [7] (Figure 2 and Additional file 1). Divergence analysis shows that four of the 3FTx groups had relatively low intra-divergence levels. Three of the groups-long chain $\alpha$-ntx (also together with kappa-ntx, $69.2 \%$ of total 3FTx ESTs in B. multicinctus), cytotoxin (also together with orphan XV group, $74.5 \%$ of total 3FTx ESTs in $N$. atra), and short chain $\alpha$-ntx (19.2\% of total 3FTx ESTs in $N$. atra)-were much more abundant than the rest of the 3FTxs (Figure 1). The other group, orphan gourp II (2.5\% of total 3FTx ESTs in B. multicinctus), have 10 cysteines and thus could be considered as an ancestral-like 3FTx group.

If the nonsynonymous to synonymous substitution rate $(d N / d S)$ for one protein-coding sequence is significantly higher than 1, it indicates a trend toward accelerated evolution. Because short coding sequences of 3FTxs have high variation (fast adaptive evolution) with multiple indels or even frameshifts between different 3FTx toxin groups, this maybe led to inaccurate multiple alignments and ambiguous phylogenetic relationships. So, we only computed $d N / d S$ rates within each of the four major 3FTx groups using PAML [19]. We observed that many $d N / d S$ values are large than 1 (Table 2). As an extreme example, we noticed that the two dominating 3FTx contigs (na001 with 227 ESTs, na054 with 310 ESTs) occupied $65.6 \%$ of all toxin ESTs in N. atra, and all of the 15 differences between these two cytotoxin contigs were

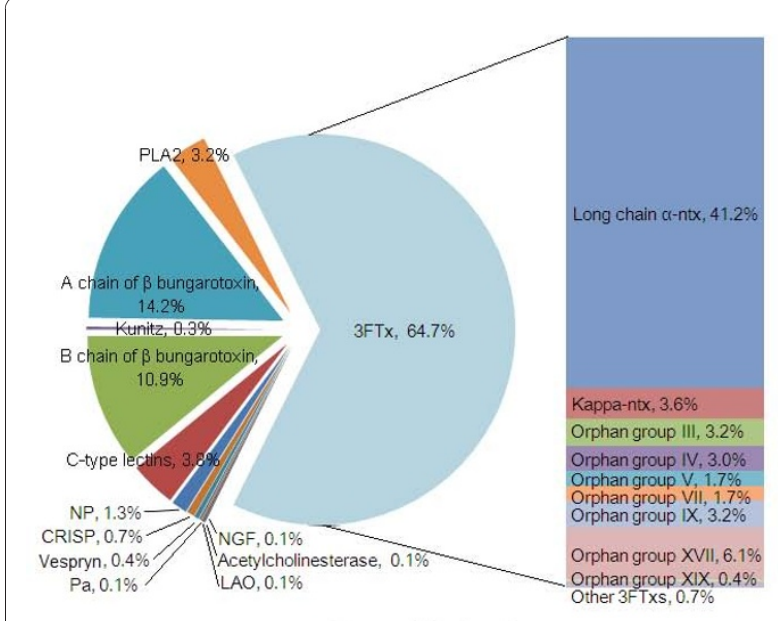

B. multicinctus

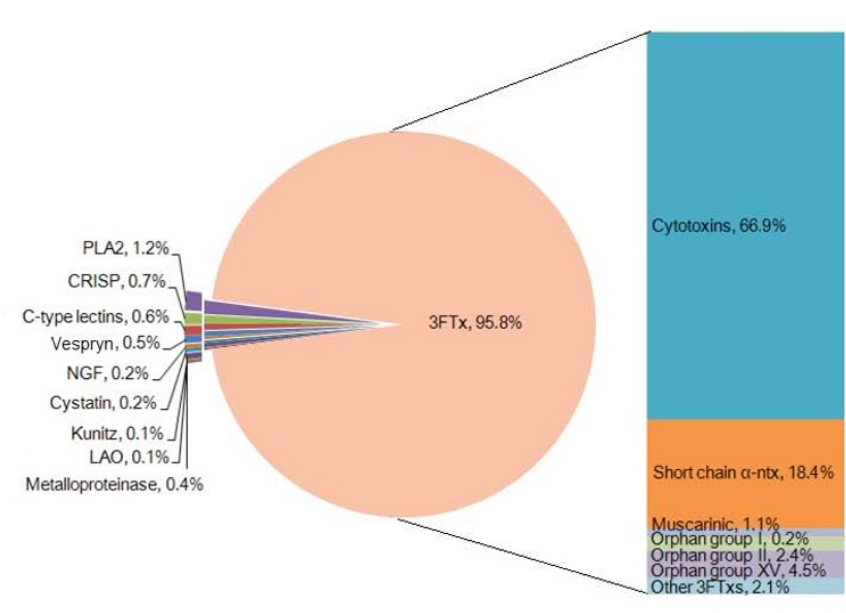

N. atra

Figure 1 Distribution of toxins in the venom gland transcriptomes of $B$. multicinctus and $N$. atra. 


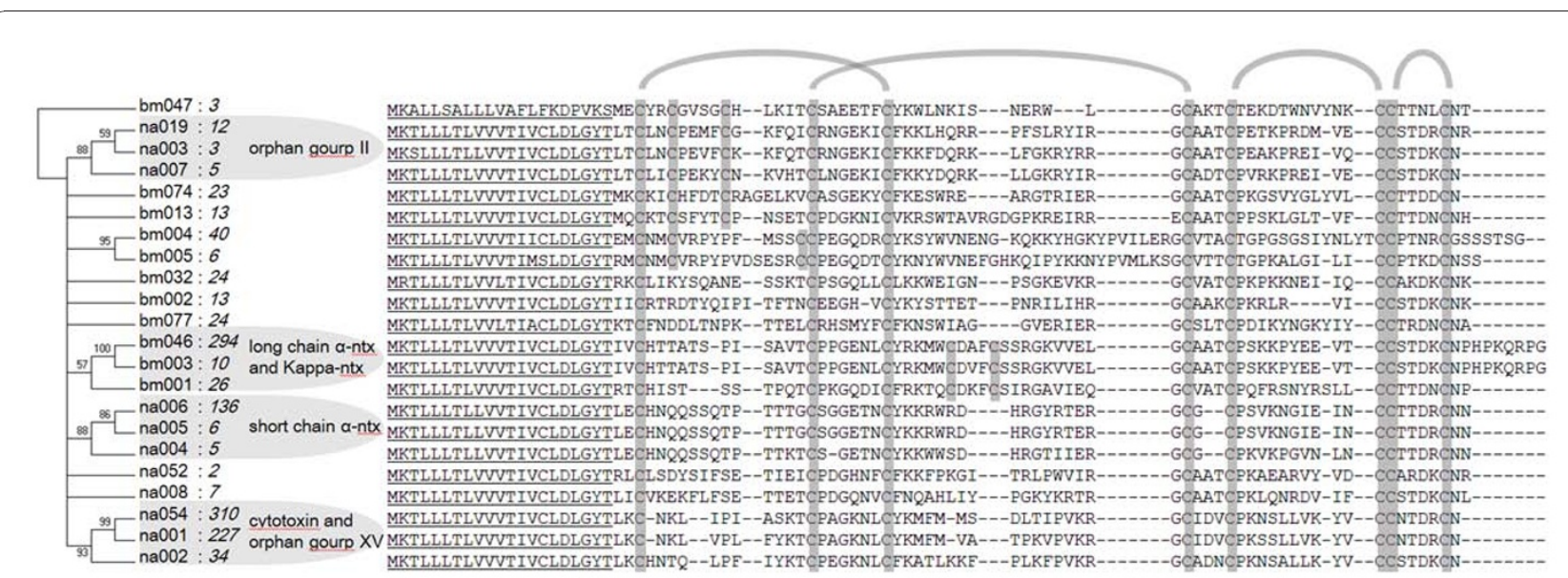

Figure 2 Representative alignments of translated $3 \mathrm{FTx}$ contigs from $\boldsymbol{B}$. multicinctus and $\mathbf{N}$. atra. Sequences can be organized in different groups, as revealed by a neighbor-joining tree on the left. Only branches with $50 \%$ or greater bootstrap support are shown. The number of ESTs is shown in italics, and the predicted signal peptide is underlined.

non-synonymous substitutions, which is significantly different from neutral expectation ( $p=0.008$, one-tail test) by Fisher's Exact Test [20], suggesting a recently accelerated diversification in this major group of 3FTxs in $N$. atra.

We also noticed a special contig, bm047 (a homologous protein previously isolated in Bungarus candidus by Kuhn et al. [21], named bucandin), which was conspicuously different from other contigs and even had a unique signal peptide (Figure 2). Based on the phylogenetic analysis, contig bm047 might have diverged from other 3FTx genes at an early stage of the origin of snakes (data not shown). It is possible that bm047 has a conserved function and has not diversified into a multicopy gene family.

\section{Phospholipase A2 and A chain of $\beta$ bungarotoxin (PLA2)}

Elapidae venom PLA2 exerts a multiplicity of novel, nonenzymatic activities, including neurotoxic and antiplatelet activity (group I), whereas viperid venom PLA2 is synovial-type (group II) PLA2 [22]. The elapid PLA2, which has 120 amino acids and 6 or 7 disulfide bonds, was thought to be derived independently from nontoxic pancreatic-type PLA2 [23].

We identified four clusters of PLA2-like ESTs with intact coding sequence (CDS) in B. multicinctus and one full-length cluster in $N$. atra, which covered the major PLA2 types detected before in B. multicinctus and $N$. atra. In B. multicinctus, one cluster (bm008) enclosing 21 ESTs showed 100\% identity with the PLA2 sequence of $B$. multicinctus in GenBank (X53406.1). Another three clusters in $B$. multicinctus were similar to the A chain of $\beta$ bungarotoxins, with a notable substitution at residue 99 (from Leu to Cyc) compared with cluster bm008, which may be important for the formation of interchain disulfides in $\beta$ bungarotoxin. Based on the gene tree, it is clear that A chain of $\beta$ bungarotoxin in B. multicinctus quite possibly duplicated from the PLA2 gene in $B$. multicinctus. We estimated an average $d S$ value of 0.27 between PLA2 contig bm008 and the A chain of $\beta$ bungarotoxins, which is smaller than the $d S$ value $(0.29)$ between bm008 and the $N$. atra PLA2 na022, indicating that the A chain of $\beta$ bungarotoxin might have derived from PLA2 after the divergence of Bungarus and Naja.

\section{Kunitz-type protease inhibitor and $B$ chain of $\beta$ bungarotoxin (Kunitz)}

Kunitz belongs to the superfamily of bovine pancreatic trypsin-like inhibitors (BPTIs), with the ancestral function of inhibiting a diverse array of serine proteases and with a peptide chain of around 60 amino acid residues and 3 disulfide bonds [3,24]. It evolved the toxin ability to inhibit various physiological processes, such as blood coagulation, fibrinolysis, host defense, and action potential transduction. In elapid venoms, besides the common Kunitz with trypsin or chymotrypsin inhibitor activity, the dendrotoxins in Dendroaspis have presynaptic neurotoxicity [25], and the $\mathrm{B}$ chain of $\beta$ bungarotoxin is thought to act as an affinity probe to guide the A chain to its target [14].

In B. multicinctus, four clusters (83 ESTs) of the B chain of a $\beta$ bungarotoxin-like sequence and one partial kunitz-type protease inhibitor-like cluster (2 ESTs) were obtained. In $N$. atra, only one singlet of a kunitz-type protease inhibitor-like EST was detected. To understand the evolutionary relationship of the kunitz-type protease inhibitor and $\mathrm{B}$ chain of $\beta$ bungarotoxin, we constructed a phylogenetic tree (Additional file 2: Figure S1). We also obtained an average $\sim 0.3 d S$ value between the kunitz- 
Table 2 Likelihood ratio test of positive selection in toxins

\begin{tabular}{|c|c|c|c|c|c|c|c|c|}
\hline $\begin{array}{l}\text { gene } \\
\text { group }\end{array}$ & $n$ & Lc & $S$ & model & $\begin{array}{l}\text { M2a } \\
\text { model } \\
\omega\left(p_{1}\right)\end{array}$ & $\begin{array}{l}\text { M8 } \\
\text { model } \\
\omega\left(p_{1}\right)\end{array}$ & $\begin{array}{l}\text { M1a- } \\
\text { M2a } \\
2 \Delta \operatorname{lnL}\end{array}$ & $\begin{array}{l}\text { M7-M8 } \\
2 \Delta \operatorname{lnL}\end{array}$ \\
\hline orphan II & 4 & 67 & 0.97 & 1.06 & $\begin{array}{l}6.56 \\
(0.102)\end{array}$ & $\begin{array}{l}6.57 \\
(0.102)\end{array}$ & 4.98 & 2.54 \\
\hline $\begin{array}{l}\text { long } \\
\text { chain }\end{array}$ & 4 & 72 & 2.43 & 2.41 & $\begin{array}{l}8.56 \\
(0.547)\end{array}$ & $\begin{array}{l}8.55 \\
(0.551)\end{array}$ & $38.98^{* *}$ & $19.47^{* *}$ \\
\hline $\begin{array}{l}\text { short } \\
\text { chain }\end{array}$ & 4 & 64 & 1.09 & 1.37 & $\begin{array}{l}5.69 \\
(0.309)\end{array}$ & $\begin{array}{l}4.21 \\
(0.461)\end{array}$ & $7.77^{*}$ & 5.81 \\
\hline cytotoxin & 4 & 64 & 1.53 & 2.66 & $\begin{array}{l}19.5 \\
(0.505)\end{array}$ & $\begin{array}{l}19.5 \\
(0.505)\end{array}$ & $26.52^{* *}$ & $17.25^{* *}$ \\
\hline $\begin{array}{l}\text { PLA2 } \\
\text { (only) }\end{array}$ & 4 & 125 & 1.66 & 0.82 & $\begin{array}{l}3.16 \\
(0.362)\end{array}$ & $\begin{array}{l}3.01 \\
(0.391)\end{array}$ & $13.78^{* *}$ & $6.91^{*}$ \\
\hline PLA2 & 6 & 125 & 2.43 & 0.95 & $\begin{array}{l}4.40 \\
(0.300)\end{array}$ & $\begin{array}{l}4.34 \\
(0.306)\end{array}$ & $30.24^{* *}$ & $15.74^{* *}$ \\
\hline lectins & 7 & 134 & 3.67 & 1.25 & $\begin{array}{l}3.36 \\
(0.341)\end{array}$ & $\begin{array}{l}3.32 \\
(0.352)\end{array}$ & $27.26^{* *}$ & $14.30^{* *}$ \\
\hline Bbeta & 4 & 61 & 1.73 & 0.80 & $\begin{array}{l}10.8 \\
(0.200)\end{array}$ & $\begin{array}{l}10.8 \\
(0.200)\end{array}$ & $25.18^{* *}$ & $12.73^{* *}$ \\
\hline Kunitz & 3 & 58 & 0.87 & 2.49 & $\begin{array}{l}19.7 \\
(0.312)\end{array}$ & $\begin{array}{l}18.3 \\
(0.318)\end{array}$ & $18.98^{* *}$ & $11.90^{* *}$ \\
\hline LAO & 5 & 493 & 0.60 & 1.18 & $\begin{array}{l}3.67 \\
(0.294)\end{array}$ & $\begin{array}{l}3.67 \\
(0.293)\end{array}$ & $29.13^{* *}$ & $14.58^{* *}$ \\
\hline Metal & 8 & 605 & 2.52 & 1.06 & $\begin{array}{l}4.30 \\
(0.227)\end{array}$ & $\begin{array}{l}4.23 \\
(0.238)\end{array}$ & $179.63^{*}$ & $100.69^{* *}$ \\
\hline CRISP & 8 & 221 & 1.46 & 1.19 & $\begin{array}{l}3.69 \\
(0.308)\end{array}$ & $\begin{array}{l}3.70 \\
(0.308)\end{array}$ & $36.62^{* *}$ & $18.39^{* *}$ \\
\hline NP & 6 & 50 & 5.17 & 0.48 & $\begin{array}{l}10.6 \\
(0.122)\end{array}$ & $\begin{array}{l}9.76 \\
(0.122)\end{array}$ & $11.91^{* *}$ & 5.54 \\
\hline NGF & 9 & 117 & 0.94 & 1.41 & $\begin{array}{l}8.08 \\
(0.151)\end{array}$ & $\begin{array}{l}7.84 \\
(0.154)\end{array}$ & $39.94^{* *}$ & $19.96^{* *}$ \\
\hline vespryn & 7 & 159 & 0.30 & 0.87 & $\begin{array}{l}3.61 \\
(0.273)\end{array}$ & $\begin{array}{l}3.61 \\
(0.273)\end{array}$ & $6.66^{*}$ & 3.34 \\
\hline
\end{tabular}

Note: $n$ represents number of sequences in a gene group. $L c$ represents number of codons in the gene after alignment gaps are removed. $S$ is the tree length, measured asqqqq the number of nucleotide substitutions per codon. $\omega$ is the estimate of the $\mathrm{dN} / \mathrm{dS}$ ratio under the M0, M2a or M8 model [19]. The proportions of sites under positive selection $\left(p_{1}\right)$ are given under the $\mathrm{M} 2 \mathrm{a}$ or $\mathrm{M} 8$ model. The $2 \mathrm{AlnL}$ parameter in the tests of M1a versus M2a or M8 versus $M 7$, indicating positive selection is marked with *. *: significant at $5 \%$ level; **: significant at $1 \%$ level. PLA2 (only) $=$ Phospholipase A2, Bbeta $=\mathrm{B}$ chain of $\beta$ bungarotoxin, Kunitz = Kunitz-type protease inhibitor, Metal = Metalloproteinase.

type protease inhibitor and $\mathrm{B}$ chain of $\beta$ bungarotoxin. Compared with the similar $d S$ value of 0.27 between PLA2 contig bm008 and A chain of $\beta$ bungarotoxin, we propose that the $A$ chain and $B$ chain of $\beta$ bungarotoxin evolved separately from PLA2 and Kuntiz around the same time as the common ancestor of Bungarus.

\section{C-type lectin}

C-type lectins or C-type lectin-like proteins are ubiquitous components of animal venoms and contain distinct diversified subgroups, including true lectins, coagulant proteins, platelet aggregation agonists, and platelet aggregation antagonists [26]. The elapid C-type lectins are arranged inside the true lectins group, except one $M$. corallinus $C$ type lectin [12]. The true lectins cause aggregation of erythrocytes in a dose-dependent manner, which are normally composed of two covalently linked identical subunits, each consisting of 135-141 amino acids [26].

Here, 29 C-type lectins-like ESTs were grouped into 9 clusters (4 contigs and 5 singlets) in B. multicinctus, while only one contig from 5 ESTs was identified in $N$. atra. Interestingly, all of these clones do not match the previously sequenced C-type lectins in B. multicinctus and $N$. atra. Phylogenetic analysis revealed that a cluster of three contigs (bm016, bm017, bm018) that we sequenced were grouped separately from all of the known Bungarus lectins, indicating that this is a novel cluster that diverged from the main group (Additional file 2: Figure S2).

\section{Metalloproteinase}

Metalloproteinase is the dominant component in viperid venoms, which exerts anticoagulant or coagulant effects and results in a severe bleeding by interfering with blood coagulation and hemostatic plug formation or by degrading the basement membrane or extracellular matrix components of the victims [27]. But, few metalloproteinases are detected in elapids [28], which produce long cDNA products (about $1.8 \mathrm{~kb}$ ). Here, three partial singlet ESTs in $N$. atra represented two kinds of metalloproteinase isoforms, which were respectively similar to EF080840 in N. atra and AY101383 in Naja mossambica as P-III type metalloproteinases.

\section{Natriuretic peptide (NP)}

The NP family functions to control natriuresis, diuresis, blood pressure, homeostasis, and inhibition of aldosterone secretion in all vertebrates or is used by snakes to interrupt these physiological processes of preys $[29,30]$ and thus has attracted attention as ideal candidates of hypotensive and vasodilator agents. It has a conserved ring structure, consisting of 17 amino acids, with extensions of a few amino acids in the two termini. Four major members of the NP family have been indentified in vertebrates-atrial NP (ANP), B-type NP (BNP), C-type NP (CNP), and ventricular NP (VNP)-and structurally, CNP lacks the C-terminal tail, whereas other NPs have both tails $[29,31]$. All of the snake venom NPs seem to have originated from a common ancestral venom, CNP, and a complete snake CNP pro-peptide is constituted by a signal peptide, a linker, a flexible $\mathrm{N}$-terminal extension (also named the CNP-53 isoform, with a 17-aa core ring), and a mature peptide (CNP-22). Nevertheless, snake NPs have recruited some new segments, such as several bradykinin-potentiating peptides (BPPs), located in the NP precursor in the Viperidae family, whereas an alterable $\mathrm{C}$-terminal extension was acquired in the Elapidae family, which may result in novel physiological actions $[13,32,33]$.

Here, we detected one contig (bm026 with 10 ESTs) only in B. multicinctus, and this transcript had a region 


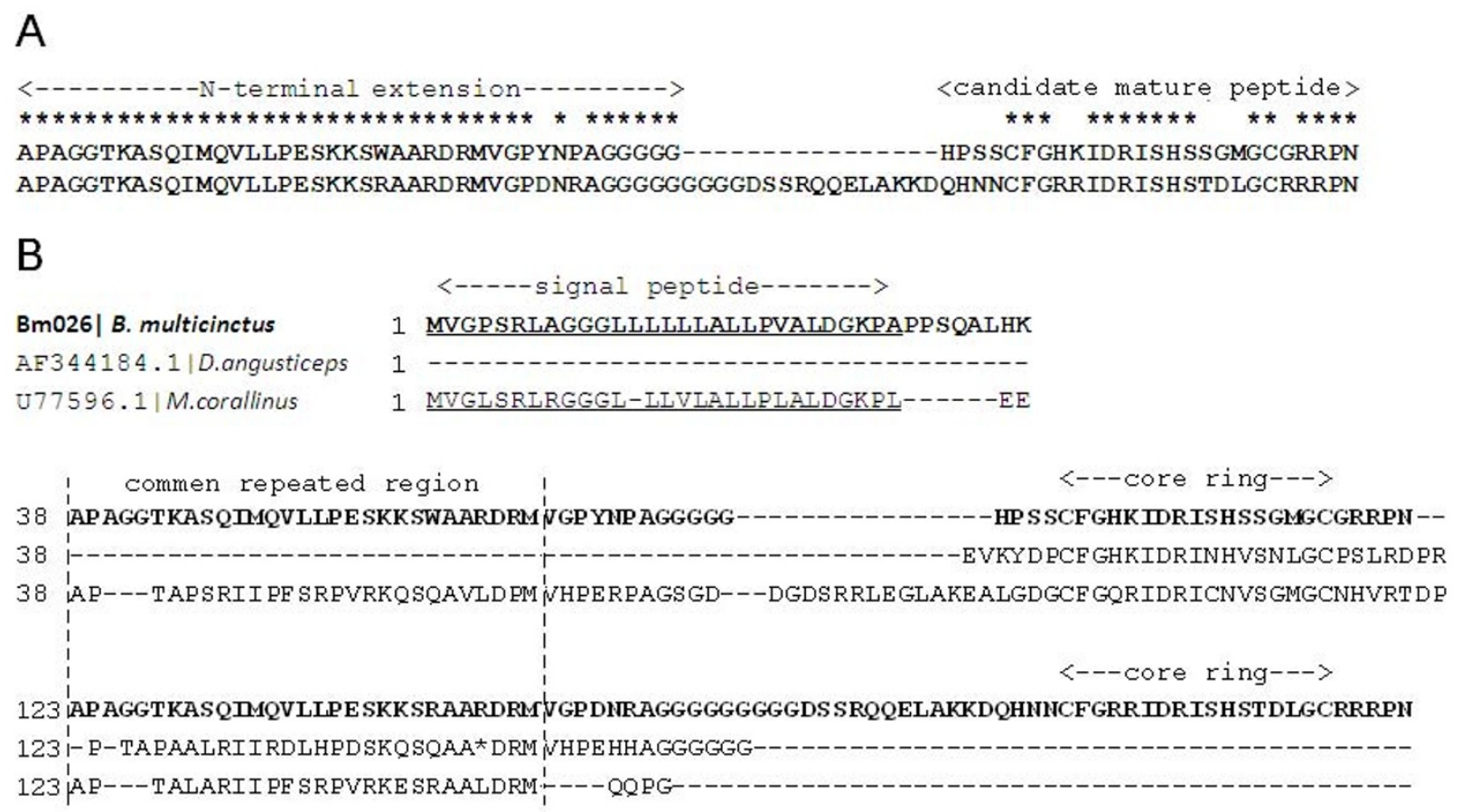

Figure 3 Alignments of natriuretic peptide (NP) sequences. (A) Alignment between putative peptides of contig bm026 (38-104) and bm026 (105-187), and the conserved amino acids are indicated by *. The predicted regions of the $\mathrm{N}$-terminal extension and mature natriuretic peptide are indicated above the alignment. (B) Bm026, the NP precursor in B. multicinctus (bold), is aligned to other elapid NP sequences. A repeated region is shown between dashed bars, and the predicted regions of signal peptide and core ring are indicated above the alignment.

containing two tandem CNP-53 repeats. We noticed that this pair of tandem duplicates had different putative mature peptides (10 differences in a 69-bp sequence) but had almost identical N-terminal extensions (3 differences in a 123-bp sequence) (Figure 3A), with statistically significant region-biased mutations ( $p=0.006$, two-tail test) by Fisher's Exact Test, which may represent a functional differentiation between the two NPs. Two possible evolutionary scenarios can explain this pattern: a) this is an old duplicate event, but the $\mathrm{N}$ terminal extensions have a recent conversion or may be under strong functional constraint; and b) this is a young duplicate event, and the putative mature peptides evolved very fast. However, based on the alignment of NP sequences in Elapidae venoms (Figure 3B), we noticed that bm026 and another unusual NP (U77596 from M.corallinus) had a common repeated region $[12,34]$. We also found that the closest sequence of bm026 was the DNP in green mamba snake (Dendroaspis angusticeps), which has attracted attention abroad for its novel long C-terminal extension [35], and Figure $3 \mathrm{~B}$ shows that the $\mathrm{C}$-terminal extension of DNP is homologous with the common repeated region. Based on these clues, we propose that the duplicate event may have occurred in the ancestor of Bungarus, Dendroaspis, and Micrurus.

\section{Other toxins}

In the cDNA libraries, except for the major multigene toxin families, there were some other toxins that were widely found in snake venoms but that only constituted a small part of the total ESTs in a species. Most of these toxins had been recruited into the venom system before the last common ancestor of elapid and viperid snakes with assistant toxic functions or unknown functions, such as CRISP, NGF, LAO, cystatin, and acetylcholinesterase $[4,5]$. Besides, vespryn (also named Ohanin, as first detected in Ophiophagus hannah) and prothrombin activator (similar to the mammalian blood coagulation factor $\mathrm{Xa}$ ) were only found in that elapid species so far [36].

In this study, we obtained many full or partial novel sequences of these less abundant toxins: one cluster in $B$. multicinctus and three clusters in $N$. atra coding for CRISP, one cluster in B. multicinctus and one cluster in $N$. atra coding for NGF, one cluster in B. multicinctus and one cluster in $N$. atra coding for LAO, three clusters in B. multicinctus and one cluster in $N$. atra coding for vespryn, one cluster coding for cystatin, and one cluster coding for acetylcholinesterase in $N$. atra.

Comparison of the major venom components among elapid snakes

Previously several other transcriptomes of the elapid venom gland had been reported, such as marine snakes 
(Lapemis curtus and Acalyptophis peronii), Australian snake (Austrelaps labialis), and coral snake (Micrurus corallines) [10-12]. However, besides 3FTxs, PLA2 is also another major venom component in these species. We also noticed that the major types, 3FTxs, are very different. The major 3FTx types from the marine and Australian snakes are long chain $\alpha$-ntx and short chain $\alpha$-ntx, whereas Micrurus corallines mainly contains orphan gourp XII and some unknown groups of 3FTxs. In contrast, the major components of $B$. multicinctus venom transcriptome are 3FTxs (mainly belong to long chain $\alpha$-ntx group) and recently originated $\beta$ bungarotoxin while the $N$. atra venom transcriptome mainly contains cytotoxin and short chain $\alpha$-ntx groups of 3FTx. Other less abundant toxins were not observed in the transcriptomes of the marine and Australian snakes, maybe due to the small number of sequenced clones.

Besides transcripts, some studies also described peptide sequences for some elapid snake toxins. A study characterizing venom proteins of $N$. atra identified 124 protein segments or peptides [37], 74\% of which belonged to 3FTxs and $11 \%$ were PLA2. In another study of Naja kaouthia venom proteomics [38], 61 venom proteins segments or peptides were identified, most of which were covered by our EST sequence, except the cobra venom factors. Two studies on coral snakes identified 26 and 11 toxin proteins or peptides, respectively $[39,40], 3 F T x$ and PLA2 proteins were also the major toxin components. So, the major toxins are basically consistent with our results observed in the $N$. atra venom gland transcriptome while Bungarus has quite different components. However, it seems the PLA2 transcripts have higher expression efficiency, considering that the PLA2 ESTs only comprise $1.2 \%$ of our toxin ESTs in N. atra while PLA2 peptides were observed to compose of a considerable amount in the $N$. atra venom [37].

\section{Analyses on the ratios of nonsynonymous to synonymous} substitution rates $(d N / d S)$ for the protein-coding ESTs of all toxin genes

It has been known that the 3FTx and PLA2 toxin multigene families have been subjected to positive Darwinian selection $[6,41]$, but whether this has happened in the less abundant toxins is still unknown. We used the maximum likelihood model to estimate the $d N / d S$ ratios of all kinds of toxins in elapid snake venoms. The representative sequences used here were from our EST data or downloaded from the GenBank database (Additional file 2: Table S1). Except for orphan group II of 3FTxs in B. multicinctus, almost all of the toxins had some sites that showed evidence of positive selection $\mathrm{dN} / \mathrm{dS}$ ratios, and the $\mathrm{dN} / \mathrm{dS}$ ratios for these sites ranged from 3.01 to 19.52 (Table 2). These results suggest that most toxin genes in elapid venoms might have been subjected to strong pressure of prey species shifts or arsenal competition, leading to the accelerated innovation of antigenic epitopes in toxins.

BAC Library construction and sequencing of 3FTx genes In order to understand the gene structure of toxin genes, two independent BAC libraries containing 73,728 (B. multicinctus) and 82,944 (N. atra) clones, with average inserted genomic segments of 120 and $100 \mathrm{~kb}$ respectively, were constructed and arrayed into 384-well microtiter plates. Examples of 16 digested clones are presented in Additional file 2: Figure S3. We estimated that the percentage of clones without inserts was about $10 \%$. Based on the snake's haploid genome size of 2.38 pg for Bungarus fasciatus and 2.59 pg for Naja haje [42], we estimated the genome coverage of the libraries to be around $3.3 \times$ for $B$. multicinctus and $2.9 \times$ for $N$. atra. These coverages theoretically provide $97.6 \%$ and $96.0 \%$ probability of obtaining any unique sequence in the B. multicinctus and $N$. atra BAC libraries, respectively, assuming random cloning [43].

We used 3FTx, PLA2, Kunitz, or NP toxin probes to screen the two BAC libraries and identified 50 positive BAC clones for $B$. multicinctus and 21 for $N$. atra. The screening results are listed in Table 3 . Unfortunately, we did not find positive PLA2 clones for $N$. atra. In addition, we identified positive NP BAC clones in N. atra, but we did not observe ESTs from its cDNA library.

In order to understand evolution patterns in the 3FTx super-family, we tried to subclone and sequence the

Table 3 Number of positive toxin BAC clones identified by different toxin probes

\begin{tabular}{|c|c|c|c|c|}
\hline & \multicolumn{2}{|c|}{ number of positive toxin BAC clones } & \multicolumn{2}{|c|}{ probe types used in blot screening } \\
\hline & B. multicinctus & N. atra & B. multicinctus & N. atra \\
\hline \multirow[t]{4}{*}{$3 F T x$} & 23 & 16 & 3FTx long chain $\alpha$-ntx & 3FTx short chain $\alpha$-ntx \\
\hline & & & 3FTx kappa-ntx & 3FTx cytotoxin \\
\hline & & & 3FTx orphan group IV & 3FTx orphan group I \\
\hline & & & 3FTx orphan group IXX & 3FTx orphan group ॥ \\
\hline PLA2 & 16 & 0 & $\beta$ bungarotoxin A-chain & PLA2 \\
\hline Kuntiz & 10 & 5 & $\beta$ bungarotoxin B-chain & \\
\hline NP & 3 & 1 & $\mathrm{NP}$ & \\
\hline Sum (remove overlap clones) & 50 & 21 & Totaled with 7 probes & Totaled with 5 probes \\
\hline
\end{tabular}


3FTx toxin genes. There were 39 BAC clones showing 3FTx-probe positivity in this study. As the major toxin in elapid venoms, the structure and organization of 3FTx toxin genes have clearly been found to consist of three exons and two introns in a region of about $2.5 \mathrm{~kb}$ [18]. Based on the results of EcoR I digestion and agarose gel electrophoresis separation, most of the 39 BAC clones had multiple 3FTx toxin gene fragments, whose sizes were from $5 \mathrm{~kb}$ to $10 \mathrm{~kb}$ (Figure 4). Considering that the 3FTx toxin genes are about $2.5 \mathrm{~kb}$ long and seldom contain EcoR I sites, these multiple 3FTx toxin gene fragments, located in the same BAC clone, are very likely to be tandem duplicates.

Excluding some redundant BAC clones with the same 3FTx toxin gene restriction fragments, we obtained 25 RFLP-unique 3FTx toxin BAC clones, indicating there are at least 13 and 12 segmental duplicate areas of 3FTx toxin genes, respectively, in B. multicinctus and $N$. atra. Because of the existence of tandem duplicates, it is hard to sequence these clones. Finally, we got 18 and 22 complete or partial 3FTx toxin gene sequences by constructing subcloning libraries and genomic walking, respectively, for $B$. multicinctus and $N$. atra. The 3FTx toxin gene sequences that were derived from the same BAC clone were defined as putative tandem duplicates. There are 5 putative tandem duplicates in $B$. multicinctus and 7 in $N$. atra. The nucleotide substitution rate per site $(K n)$ in the non-coding regions (intron I and intron II of 3FTx gene) between the tandem duplicates is shown in Table 4. We noticed that most of the potential tandem duplicates had low $K n$ values $(<0.05)$, indicating that most duplication events happened recently. It also suggests that fast 3FTx gene expansion is still ongoing in the two elapids.

Based on the best Blast hits to the GenBank database and our EST data, all of the 3FTx toxin genes were classified and annotated (Additional file 2: Table S2). About $65 \%(26 / 40)$ of the gene sequences showed lower than 95\% identity with 3FTx toxin gene sequences from the GenBank database, indicating that most of the 3FTx toxin genes that we sequenced are novel sequences (Figure 5). Parts of the 3FTx genes that we sequenced, especially a big group that looked similar to orphan group II, could not match any ESTs from the two cDNA libraries, indicating that a large number of lower-expression 3FTx toxin genes were not detected in previous studies.

Because the coding sequences of 3FTx genes are short and too diversified, it is difficult to construct an accurate phylogenetic relationship for all these diverse subgroups of 3FTx. So, we tried to use only the intron sequences for genic phylogenetic analyses, especially using intron II, which has a stable length of about 550 bp [44]. But, the 3FTx gene tree (Figure 5) still confuses us with multiple radiated sub-families, just like the phylogenetic

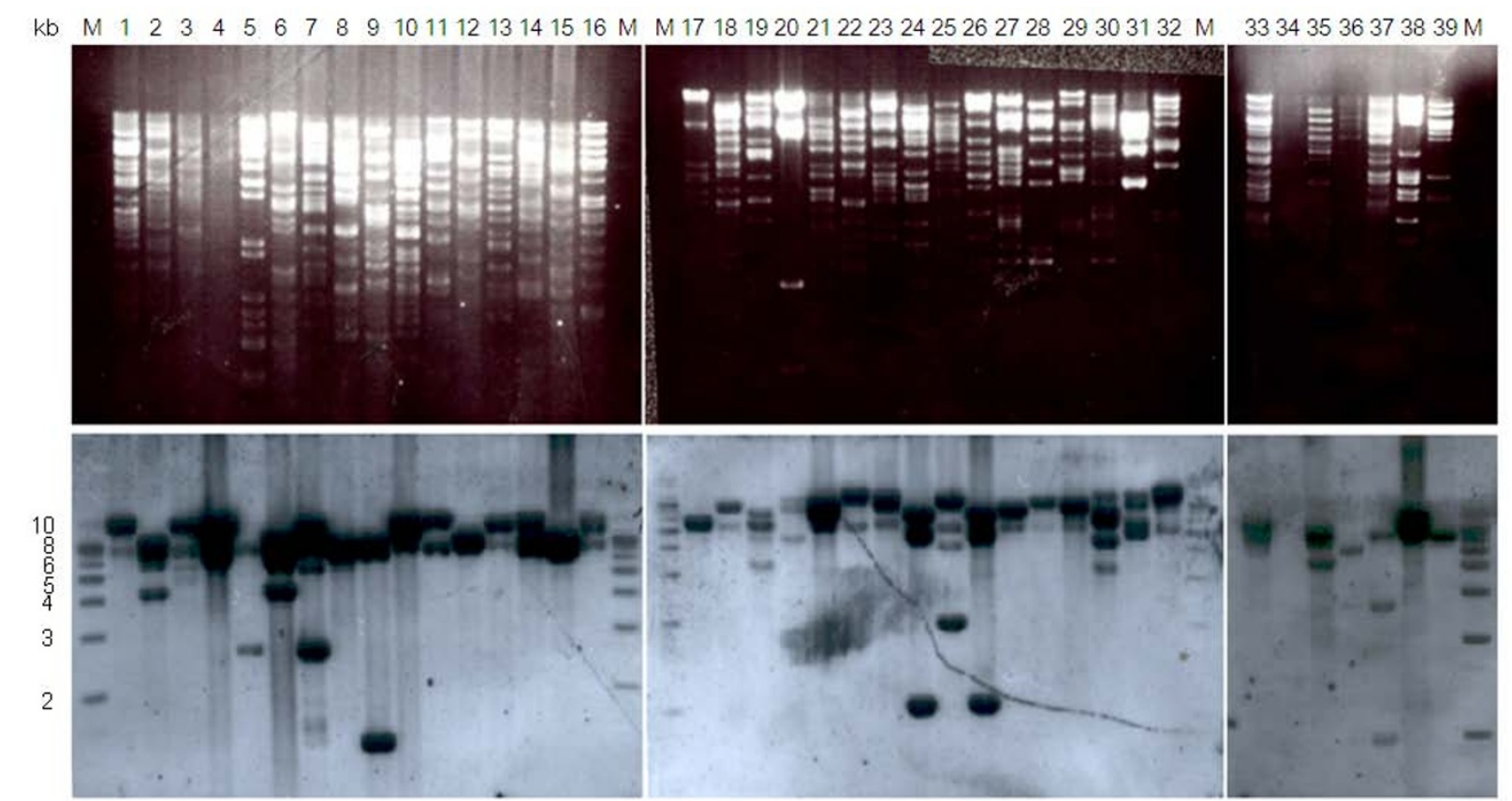

Figure 4 Blotting analysis of 39 3FTx probe-positive BAC clones. 3FTx probe-positive BAC clones were digested with EcoR I and analyzed on $0.8 \%$ agarose gels prior to blotting on a nylon membrane (upper panel). The 3FTx gene-containing fragments were identified by hybridization with digoxigenin-labeled 3FTx probes (lower panel). The positive bands were used as a control to cut and purify 3FTx genecontaining fragments in a second gel. Lanes: M, 1 kb DNA ladder; 1-39, 3FTx probe-positive BAC clones (Additional file 2: Table S2). 
Table 4 Pairwise genetic distance among putative tandem duplicates

\begin{tabular}{llll}
\hline BAC name & tandem duplicates & \multicolumn{2}{c}{ Average pairwise distance } \\
\cline { 3 - 4 } & number & Intron I & Intron II \\
\hline N044_7B & 4 & 0.018 & 0.029 \\
N072_20 m & 3 & 0.058 & 0.012 \\
N036_12b & 2 & 0.018 & 0.000 \\
N052_20J & 2 & 0.007 & 0.002 \\
N076_21 m & 2 & 0.128 & 0.014 \\
N061_6e & 2 & 0.030 & 0.002 \\
N031_7f & 2 & 0.011 & 0.002 \\
B218_4L & 2 & 0.004 & 0.002 \\
B254_9H & 2 & 0.010 & 0.007 \\
B241_M14 & 2 & 0.002 & 0.009 \\
B244_10 & 2 & 0.154 & 0.005 \\
B132_1G & 2 & - & 0.103 \\
\hline
\end{tabular}

relationship of the 3FTx constructed by Fry [4,7]. Taking $\alpha$-ntx for example, we observed two monophyletic lineages of short chain $\alpha$-ntx from $N$. atra and two monophyletic lineages of long chain $\alpha$-ntx, respectively, from $B$. multicinctus and $N$. atra, which indicates that multiple groups of $\alpha$-ntx separated before the divergence of Bungarus and Naja. Considering that the long chain $\alpha$-ntx originated mainly by one splicing site shifting event from short chain $\alpha$-ntx [18] and that both the short and long chain $\alpha$-ntx coexist in some Australian and marine snakes, such as Austrelaps labialis [11], Lapemis curtus [10], and Laticauda semifasciata [18], the hypotheses that $\alpha$-ntx diverged from a common ancestor seems reasonable. So, it is very likely that these broad radiated groups of 3FTx (containing the multiple $\alpha$-ntx, cytotoxin, and other orphan 3FTx groups) appeared right before the explosive speciation of major elapid subfamilies in the mid-Oligocene [9]. In brief, our data suggest that 3FTx genes probably experienced family explosion and thereby generated the major groups before the species radiation of Elapidae subfamilies. After that, by massive duplication, perhaps mainly by tandem duplication, the 3FTx gene family acquired numerous new members and became the most abundant toxin family in the two elapid venoms.

\section{Conclusions}

Both $B$. multicinctus and $N$. atra venoms are very toxic, respectively, with LD50 of $0.108 \mathrm{mg} / \mathrm{kg}$ and $0.29 \mathrm{mg} / \mathrm{kg}$ when injected subcutaneously (Venomdoc database from Fry), and they belong to two closely related genera [9]. However, the major components of the B. multicinctus venom transcriptome are neurotoxins, including long chain $\alpha$-ntx and $\beta$ bungarotoxin, whereas the $N$. atra venom transcriptome mainly has cytotoxicity and a little bit of neurotoxicity from short chain $\alpha$-ntx. In this study, we present the toxin profiles of $B$. multicinctus and $N$. atra by sequencing their venom gland transcriptomes. Then, the BAC libraries for these two elapids were constructed, and 25 BACs containing 3FTx genes were partially sequenced. The data revealed that tandem duplications contributed the majority of the expansions of toxin multigene families in the two elapids. We detected positive selection in every toxin subfamily and found that not only the multigene toxin families but also the less abundant toxins were under rapid adaptive evolution.

\section{Methods \\ Materials}

Fresh venom glands and blood cells were obtained, respectively, from an individual of Bungarus multicinctus and Naja atra, both of which were collected from Zhejiang province, China. The protocol was approved by the ethics committee of Kunming Institute of Zoology, CAS, China.

\section{cDNA library construction and sequencing}

Total RNA was extracted from venom glands using the RNeasy Mini Kit (Qiagen, Germany). The mRNA was purified from total RNA, using the Oligotex mRNA kit (Qiagen). The purified mRNA was used to make cDNA library, following the instructions in the SuperScript Plasmid System for cDNA Synthesis and Cloning Kit (plasmid used: pCMV-SPORT6 for B. multicinctus, pSPORT1 for $N$. atra) (Invitrogen, USA). Plasmids were purified using the QIAprep spin miniprep kit (Qiagen). Purified plasmids were sequenced by cycle sequencing reactions using the BigDye Terminator v3.1 kit (Applied Biosystem, USA) and an automated DNA sequencer (Model 3100A, Applied Biosystem, USA).

\section{cDNA sequence cluster assembly}

After removing the vector, adaptors, and low-quality sequences, ESTs were assembled into contiguous clusters using the CAP3 program [45], with the setting that only joined sequences with at least 95\% identity. Each cluster (contigs with more than one EST or singlets with one EST) was then searched against the GenBank databases using BLASTX and BLASTN algorithms to identify similar sequences with an e-value cutoff $<10^{-5}$, as described by Ching AT et al. [13]. The signal peptide was predicted using the local SignalP 3.0 server [46]. A final annotation table was generated in Microsoft Excel format, containing all the relevant information about clusters and singlets. EST sequences were deposited in GenBank dbEST under accession numbers HO056271 to HO058536.

\section{Bacterial artificial chromosome (BAC) library construction and characterization}

High-molecular-weight DNA was extracted from snake blood cells, partially digested with EcoR I, and cloned 


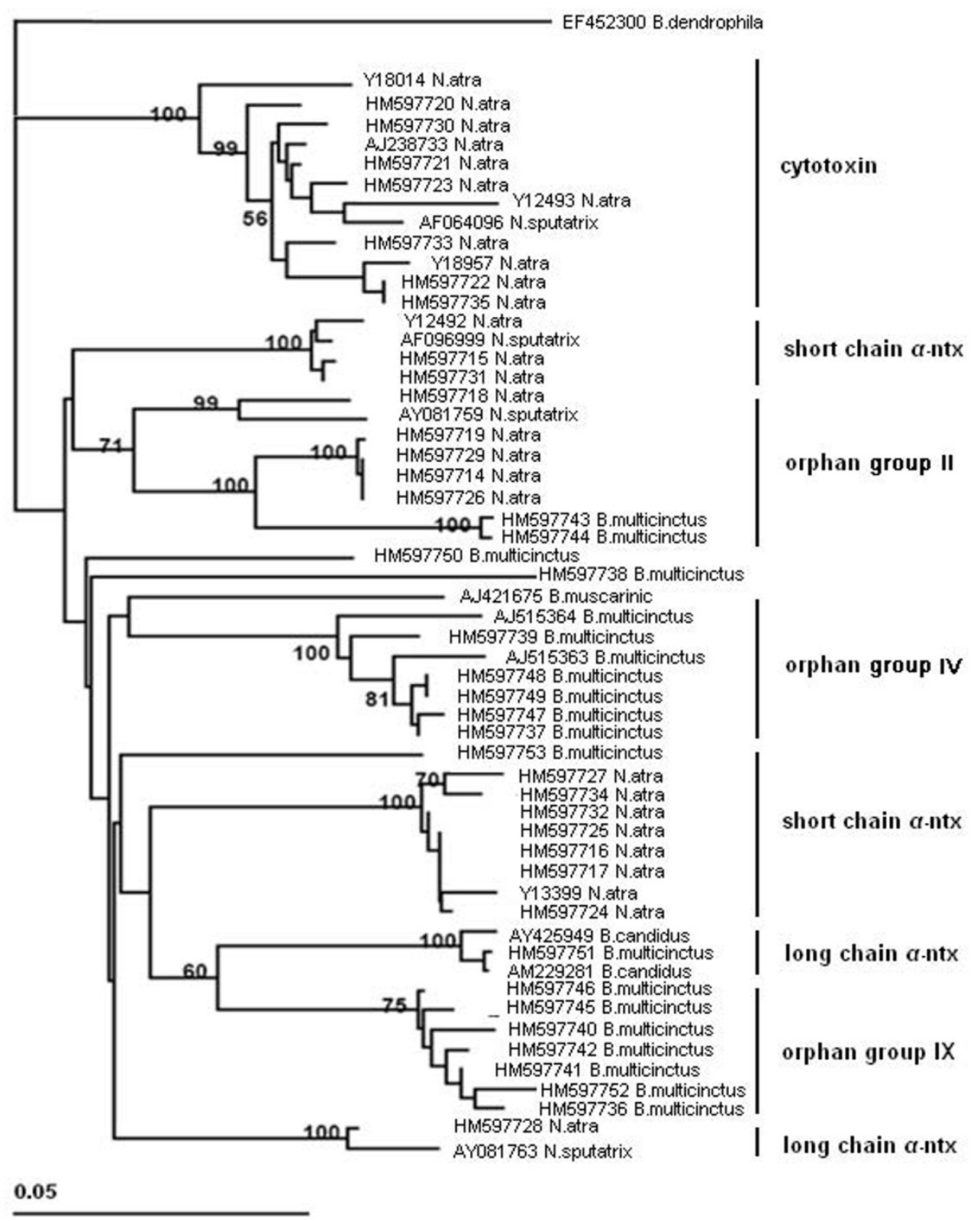

Figure 5 Phylogenetic tree constructed using 3FTx intron II sequences with the maximum likelihood method in phylip. The intron sequences were from our BAC libraries and the GenBank database, both in the genus of Bungarus and Naja, using a colubrid (Boiga dendrophila) 3FTx as the outgroup. Numbers above branches indicate posterior probability values higher than $50 \%$. Sequence names are labeled according the GenBank Accn. Number, followed by the species they were identified from. The sequences are classified basically based on the CDS,

following the nomenclature of Fry [7].

into the CopyControl pCC1BAC vector (Epicentre, USA) according to a previously described method [47]. To estimate the recombination ratio of the libraries and the average size of inserted DNA fragments, 100 randomly selected BAC clone DNAs were digested with Not I and separated by PFGE, as described by Wang et al. [48].

\section{Screening the snake BAC genomic library}

All the colonies in 384-well microtiter plates were replica-plated onto nylon membranes for hybridization using standard techniques [49]. Two rounds of screening were performed. In the first one, a mixture of 12 probes was used, which represented four kinds of toxin genes. These probes were respectively prepared for the 
consensus toxin coding sequences by labeling their PCR product with digoxigenin. They were designed based on the cDNA sequences of 3FTx, $\beta$ bungarotoxin A-chain (or PLA2), $\beta$ bungarotoxin B-chain, and NP (Additional file 2: Table S3). Then, the 79 positive clones were respectively hybridized with each kind of probe to exclude false positives and identify the concrete toxin gene type.

\section{Subcloning 3FTx genes and sequencing}

All 39 3FTx gene-positive clones, verified by colony hybridization, were digested with $E c o$ R I and analyzed on $0.8 \%$ agarose gels prior to blotting on a nylon membrane. Then, 3FTx gene-containing fragments were identified by hybridization with the same probes used in the library screening and subcloned into the CopyControl pCC1BAC vector. These subclones were then tested by PCR using specific primers, designed based on the sequence of the toxin ESTs (Additional file 2: Table S3). PCR amplifications were then ligated into the pMD18-T vector (TaKaRa, Dalian), and the fragments were sequenced using an ABI 3100A Genetic Analyzer. The sequences obtained from the same subclone were assembled into error-free consensus sequences using SeqMan II software (DNAStar, Version 6.0). These sequences were compared with our ESTs and published sequences in the GenBank database using the BLASTn program. Exon-intron structures of genes were doublechecked using the Sim4 Program for spliced alignments [50].

\section{Evolutionary analyses}

DNA or protein sequences were aligned with the MUSCLE program [51] with manual adjustments. The number of nucleotide substitutions per site $(K n)$ in the noncoding regions (introns) was estimated using the Kimura 2-parameter model, and the numbers of nucleotide substitutions per synonymous site $(d N)$ and per nonsynonymous $(d S)$ in the putative protein-coding regions were computed for pairs according to the method of the realistic evolutionary model in PAML [52]. Neighbor-joining (NJ) trees were constructed using the software MEGA version 4 [53], and the maximum likelihood (ML) trees were constructed using phylip-3.68 [54], evaluated by 1000 bootstrap replications, considering ML bootstrap proportions $>70 \%$ to indicate significant support, with transition-transversion ratios estimated from the data (implemented in tree-puzzle-5.2 [55]). There are several inframe indels in the coding region of ESTs, and those sites were removed in the sequence analysis.

In order to detect selection in a toxin subfamily, the likelihood ratio test (LRT), implemented in PAML4.2, was employed to detect positive selection using both the tests of M1a versus M2a and M8 versus M7 comparisons

with the phylogeny-based approach $[19,56]$. The putative coding sequences of ESTs that showed high divergence from the other sequences in a sub-family were removed from the analysis.

\section{Additional material}

Additional file 1: The complete list of all EST clusters in $B$.
multicinctus and N. atra, annotated by using BLAST program

Additional file 2: Figure S1 and S2. Phylogenetic trees constructed, respectively, using Kunitz and lectins sequences with the maximum likelihood method in MEGA. Figure S3. Characterization of the snake BAC libraries. Sixteen randomly selected B. multicinctus and N. atra BAC clones were digested with Not I and separated by PFGE. Lane M: Lowrange PFG marker. Table S1. The representative sequences used for estimation of the $\mathrm{dN} / \mathrm{dS}$ ratios. Table S2. The detailed information of all 71 positive toxin BAC clones from the toxin probe screening. Table S3. The toxin ESTs used as hybridization probes to screen for venom genes.

\section{Acknowledgements}

We thank Xin Li and Qi Zhou for helpful discussions. This work was supported by the 100 Talents Program of Chinese Academy of Sciences and a CAS-Max Planck Society Fellowship to WW; and grants from the 973 Program (2010CB529800) and the Chinese National Natural Science Foundation (30630014) to YZ.

\section{Author details}

CAS-Max Planck Junior Research Group, State Key Laboratory of Genetic Resources and Evolution, Kunming Institute of Zoology, Chinese Academy of Sciences, Kunming, Yunnan 650223, China. ${ }^{2}$ Graduate University of Chinese Academy Sciences, Beijing 100039, China. ${ }^{3}$ College of Animal Science and Technology, Sichuan Agricultural University, Sichuan 625014, China. ${ }^{4}$ Biotoxin Units, Key Laboratory of Animal Models and Human Disease Mechanisms, Kunming Institute of Zoology, Chinese Academy of Sciences, Kunming, Yunnan 650223, China.

\section{Authors' contributions}

$Y L, X X, Y Z$, and RZ constructed the CDNA and BAC libraries. $Y J$ and $W L$ analyzed the data and wrote the manuscript. $Y Z$ and $W W$ are the principal investigators who conceived the study and critically reviewed the manuscript. All authors read and approved the final manuscript.

Received: 3 July 2010 Accepted: 3 January 2011

Published: 3 January 2011

\section{References}

1. Calvete JJ, Sanz L, Angulo Y, Lomonte B, Gutierrez JM: Venoms, venomics, antivenomics. FEBS Lett 2009, 583(11):1736-1743.

2. Fry BG, Vidal N, Norman JA, Vonk FJ, Scheib H, Ramjan SF, Kuruppu S, Fung $\mathrm{K}$, Hedges SB, Richardson MK, et al: Early evolution of the venom system in lizards and snakes. Nature 2006, 439(7076):584-588.

3. Fry BG, Scheib H, van der Weerd L, Young B, McNaughtan J, Ramjan SF, Vidal N, Poelmann RE, Norman JA: Evolution of an arsenal: structural and functional diversification of the venom system in the advanced snakes (Caenophidia). Mol Cell Proteomics 2008, 7(2):215-246.

4. Fry BG, Vidal N, van der Weerd L, Kochva E, Renjifo C: Evolution and diversification of the Toxicofera reptile venom system. J Proteomics 2009, 72(2):127-136.

5. Fry BG: From genome to "venome": molecular origin and evolution of the snake venom proteome inferred from phylogenetic analysis of toxin sequences and related body proteins. Genome Res 2005, 15(3):403-420.

6. Kordis D, Gubensek F: Adaptive evolution of animal toxin multigene families. Gene 2000, 261(1):43-52.

7. Fry BG, Wuster W, Kini RM, Brusic V, Khan A, Venkataraman D, Rooney AP: Molecular evolution and phylogeny of elapid snake venom three-finger toxins. J Mol Evol 2003, 57(1):110-129. 
8. Vonk FJ, Admiraal JF, Jackson K, Reshef R, de Bakker MA, Vanderschoot K, van den Berge I, van Atten M, Burgerhout E, Beck A, et al: Evolutionary origin and development of snake fangs. Nature 2008, 454(7204):630-633.

9. Kelly C, Barker N, Villet M, Broadley D: Phylogeny, biogeography and classification of the snake superfamily Elapoidea: a rapid radiation in the late Eocene. Cladistics 2009, 25(1):38-63.

10. Pahari S, Bickford D, Fry BG, Kini RM: Expression pattern of three-finger toxin and phospholipase A2 genes in the venom glands of two sea snakes, Lapemis curtus and Acalyptophis peronii: comparison of evolution of these toxins in land snakes, sea kraits and sea snakes. BMC Evol Biol 2007, 7:175

11. Doley R, Tram NN, Reza MA, Kini RM: Unusual accelerated rate of deletions and insertions in toxin genes in the venom glands of the pygmy copperhead (Austrelaps labialis) from Kangaroo island. BMC Evol Biol 2008, 8:70.

12. Leao LI, Ho PL, Junqueira-de-Azevedo Ide L: Transcriptomic basis for an antiserum against Micrurus corallinus (coral snake) venom. BMC Genomics 2009, 10:112.

13. Ching AT, Rocha MM, Paes Leme AF, Pimenta DC, de Fatima DFM, Serrano SM, Ho PL, Junqueira-de-Azevedo IL: Some aspects of the venom proteome of the Colubridae snake Philodryas olfersii revealed from a Duvernoy's (venom) gland transcriptome. FEBS Lett 2006, 580(18):4417-4422

14. Rowan E: What does [beta]-bungarotoxin do at the neuromuscular junction? Toxicon 2001, 39(1):107-118.

15. Pawlak J, Mackessy SP, Sixberry NM, Stura EA, Le Du MH, Menez R, Foo CS, Menez A, Nirthanan S, Kini RM: Irditoxin, a novel covalently linked heterodimeric three-finger toxin with high taxon-specific neurotoxicity. FASEB J 2009, 23(2):534-545.

16. Li J, Zhang H, Liu J, Xu K: Novel genes encoding six kinds of three-finger toxins in Ophiophagus hannah (king cobra) and function characterization of two recombinant long-chain neurotoxins. Biochem $J$ 2006, 398(2):233-242.

17. Jeyaseelan K, Poh SL, Nair R, Armugam A: Structurally conserved alphaneurotoxin genes encode functionally diverse proteins in the venom of Naja sputatrix. FEBS Lett 2003, 553(3):333-341.

18. Tamiya T, Fujimi TJ: Molecular evolution of toxin genes in Elapidae snakes. Mol Divers 2006, 10(4):529-543.

19. Yang Z: PAML 4: phylogenetic analysis by maximum likelihood. Mol Biol Evol 2007, 24(8):1586-1591.

20. Li W-H: Molecular Evolution. Sinauer Sunderland, MA; 1997

21. Kuhn P, Deacon AM, Comsa DS, Rajaseger G, Kini RM, Uson II, Kolatkar PR: The atomic resolution structure of bucandin, a novel toxin isolated from the malayan krait, determined by direct methods. erratum. Acta Crystallogr D Biol Crystallogr 2000, 56(Pt 12):1702.

22. Heinrikson RL, Krueger ET, Keim PS: Amino acid sequence of phospholipase A2-alpha from the venom of Crotalus adamanteus. A new classification of phospholipases A2 based upon structural determinants. J Biol Chem 1977, 252(14):4913-4921.

23. Fujimi TJ, Kariya Y, Tsuchiya T, Tamiya T: Nucleotide sequence of phospholipase $A(2)$ gene expressed in snake pancreas reveals the molecular evolution of toxic phospholipase A(2) genes. Gene 2002, 292(1-2):225-231.

24. Yuan C, He Q, Peng K, Diao J, Jiang L, Tang X, Liang S: Discovery of a distinct superfamily of Kunitz-type toxin (KTT) from tarantulas. PLOS One 2008, 3:10.

25. Harvey AL, Karlsson E: Protease inhibitor homologues from mamba venoms: facilitation of acetylcholine release and interactions with prejunctional blocking toxins. Br J Pharmacol 1982, 77(1):153-161.

26. Ogawa T, Chijiwa T, Oda-Ueda N, Ohno M: Molecular diversity and accelerated evolution of C-type lectin-like proteins from snake venom. Toxicon 2005, 45(1):1-14.

27. Matsui T, Fujimura Y, Titani K: Snake venom proteases affecting hemostasis and thrombosis. Biochim Biophys Acta 2000, 1477(1-2):146-156.

28. Guo XX, Zeng L, Lee $W H$, Zhang $Y$, Jin $Y$ : Isolation and cloning of a metalloproteinase from king cobra snake venom. Toxicon 2007 49(7):954-965

29. Inoue K, Takei Y: Molecular evolution of the natriuretic peptide system as revealed by comparative genomics. Comparative Biochemistry and Physiology Part D: Genomics and Proteomics 2006, 1(1):69-76.
30. St Pierre L, Flight S, Masci PP, Hanchard KJ, Lewis RJ, Alewood PF, de Jersey J, Lavin MF: Cloning and characterisation of natriuretic peptides from the venom glands of Australian elapids. Biochimie 2006, 88(12):1923-1931.

31. Trajanovska S, Donald J: Molecular cloning of natriuretic peptides from the heart of reptiles: loss of ANP in diapsid reptiles and birds. General and comparative endocrinology 2008, 156(2):339-346.

32. Baxter G: The natriuretic peptides. Basic research in cardiology 2004, 99(2):71-75.

33. Fry BG, Wickramaratana JC, Lemme S, Beuve A, Garbers D, Hodgson WC Alewood P: Novel natriuretic peptides from the venom of the inland taipan (Oxyuranus microlepidotus): isolation, chemical and biological characterisation. Biochem Biophys Res Commun 2005, 327(4):1011-1015.

34. Ho PL, Soares MB, Maack T, Gimenez I, Puorto G, Furtado MF, Raw I: Cloning of an unusual natriuretic peptide from the South American coral snake Micrurus corallinus. Eur J Biochem 1997, 250(1):144-149.

35. Schweitz $H$, Vigne $P$, Moinier D, Frelin C, Lazdunski M: A new member of the natriuretic peptide family is present in the venom of the green mamba (Dendroaspis angusticeps). J Biol Chem 1992, 267(20):13928-13932

36. Reza MA, Swarup S, Kini RM: Structure of two genes encoding parallel prothrombin activators in Tropidechis carinatus snake: gene duplication and recruitment of factor $\mathrm{X}$ gene to the venom gland. J Thromb Haemost 2007, 5(1):117-126

37. Li S, Wang J, Zhang X, Ren Y, Wang N, Zhao K, Chen X, Zhao C, Li X, Shao J, et al: Proteomic characterization of two snake venoms: Naja naja atra and Agkistrodon halys. Biochem J 2004, 384(Pt 1):119-127.

38. Kulkeaw K, Chaicumpa W, Sakolvaree $Y$, Tongtawe $P$, Tapchaisri P: Proteome and immunome of the venom of the Thai cobra, Naja kaouthia. Toxicon 2007, 49(7):1026-1041.

39. Olamendi-Portugal T, Batista CV, Restano-Cassulini R, Pando V, VillaHernandez O, Zavaleta-Martinez-Vargas A, Salas-Arruz MC, Rodriguez de la Vega RC, Becerril B, Possani LD: Proteomic analysis of the venom from the fish eating coral snake Micrurus surinamensis: novel toxins, their function and phylogeny. Proteomics 2008, 8(9):1919-1932.

40. Dokmetjian JC, Del Canto S, Vinzon S, de Jimenez Bonino MB: Biochemical characterization of the Micrurus pyrrhocryptus venom. Toxicon 2009, 53(3):375-382.

41. Lynch VJ: Inventing an arsenal: adaptive evolution and neofunctionalization of snake venom phospholipase A2 genes. BMC Evol Biol 2007, 7:2.

42. De Smet W: The nuclear Feulgen-DNA content of the vertebrates (especially reptiles), as measured by fluorescence cytophotometry, with notes on the cell and chromosome size. Acta Zool Pathol Antverp 1981, 76:119-167.

43. Clarke L, Carbon J: A colony bank containing synthetic Col El hybrid plasmids representative of the entire E. coli genome. Cell 1976, 9(1):91-99.

44. Fujimi TJ, Nakajyo T, Nishimura E, Ogura E, Tsuchiya T, Tamiya T: Molecular evolution and diversification of snake toxin genes, revealed by analysis of intron sequences. Gene 2003, 313:111-118.

45. Huang X, Madan A: CAP3: A DNA sequence assembly program. Genome Res 1999, 9(9):868-877.

46. Bendtsen JD, Nielsen $H$, von Heijne $G$, Brunak S: Improved prediction of signal peptides: SignalP 3.0. J Mol Biol 2004, 340(4):783-795.

47. Wang Z, Miyake T, Edwards SV, Amemiya CT: Tuatara (Sphenodon) genomics: BAC library construction, sequence survey, and application to the DMRT gene family. J Hered 2006, 97(6):541-548.

48. Wang $W$, Xu HL, Lin LP, Su B, Wang YQ: Construction of a BAC library for Chinese amphioxus Branchiostoma belcheri and identification of clones containing Amphi-Pax genes. Genes Genet Syst 2005, 80(3):233-236.

49. Chiu CH, Amemiya CT, Carr JL, Bhargava J, Hwang JK, Shashikant CS, Ruddle FH, Wagner GP: A recombinogenic targeting method to modify large-inserts for cis-regulatory analysis in transgenic mice: construction and expression of a 100-kb, zebrafish Hoxa-11b-lacZ reporter gene. Dev Genes Evol 2000, 210(2):105-109.

50. Florea L, Hartzell G, Zhang Z, Rubin GM, Miller W: A computer program for aligning a cDNA sequence with a genomic DNA sequence. Genome Res 1998, 8(9):967-974

51. Edgar RC: MUSCLE: multiple sequence alignment with high accuracy and high throughput. Nucleic Acids Res 2004, 32(5):1792-1797. 
52. Yang Z, Nielsen R: Estimating synonymous and nonsynonymous substitution rates under realistic evolutionary models. Mol Biol Evol 2000, 17(1):32-43.

53. Tamura K, Dudley J, Nei M, Kumar S: MEGA4: Molecular Evolutionary Genetics Analysis (MEGA) software version 4.0. Mol Biol Evol 2007, 24(8):1596-1599.

54. Felsenstein J: PHYLIP (phylogeny inference package) version 3.6. Distributed by the author Department of Genome Sciences, University of Washington, Seattle 2005

55. Schmidt H, Strimmer K, Vingron M, Von Haeseler A: TREE-PUZZLE: maximum likelihood phylogenetic analysis using quartets and parallel computing. Bioinformatics 2002, 18(3):502.

56. Yang Z, Nielsen R, Goldman N, Pedersen AM: Codon-substitution models for heterogeneous selection pressure at amino acid sites. Genetics 2000 155(1):431-449.

doi:10.1186/1471-2164-12-1

Cite this article as: Jiang et al:: Venom gland transcriptomes of two elapid snakes (Bungarus multicinctus and Naja atra) and evolution of toxin genes. BMC Genomics 2011 12:1.

\section{Submit your next manuscript to BioMed Central} and take full advantage of:

- Convenient online submission

- Thorough peer review

- No space constraints or color figure charges

- Immediate publication on acceptance

- Inclusion in PubMed, CAS, Scopus and Google Scholar

- Research which is freely available for redistribution

Submit your manuscript at www.biomedcentral.com/submit 